\title{
Time-resolved Micro-Raman Measurement of Temperature Dynamics during High-Repetition-Rate Ultrafast Laser Microprocessing
}

\author{
F. Hashimoto ${ }^{* 1}$, S. Richter ${ }^{* 2}$, S. Nolte ${ }^{* 2}$, Y. Ozeki ${ }^{* 1}$, and K. Itoh ${ }^{* 1}$ \\ ${ }^{*}$ Graduate School of Engineering, Osaka University, \\ 2-1 Yamadaoka, Suita, Osaka 565-0871, Japan \\ ${ }^{* 2}$ Institute of Applied Physics, Friedrich-Schiller-University, \\ Max-Wien-Platz 1, 07743 Jena, Germany \\ E-mail: hashimoto@photonics.mls.eng.osaka-u.ac.jp
}

\begin{abstract}
We employed time-resolved micro-Raman spectroscopy to investigate the temperature dynamics inside glass during high-repetition-rate femtosecond laser microprocessing. We measured the temperature evolutions as functions of the distance from the focus of femtosecond laser pulses, although it was difficult to acquire Raman spectra at locations near the focus due to the onset of strong background emissions. The measured temperature data were fitted by, taking the heat accumulation of successive laser pulses into account. We further investigated the dependence of temperature increase on the processing parameters. Our results suggest that the monitoring of thermal process is useful not only for process optimization but also for detailed in situ analysis of thermal process in highrepetition-rate femtosecond laser microprocessing.
\end{abstract}

DOI: $10.2961 /$ jlmn.2015.01.0006

Keywords: femtosecond laser, microprocessing, time-resolved measurement, Raman temperature measurement, thermal process, temperature dynamics, heat accumulation

\section{Introduction}

When a transparent material such as glass is irradiated by tightly focused femtosecond laser pulses, localized material modification can occur inside the material. Such modification has been utilized for the fabrication of microphotonic devices and the microwelding of transparent materials $[1,2]$. However, the mechanisms behind the process therein are still not completely understood and there is a need for quantitative investigation of the process. Among several mechanisms involved in femtosecond laser processing such as multiphoton ionization, plasma formation, thermal relaxation and its diffusion, thermal processes play important roles because they strongly affect the resulting structural change and material characteristics after processing [3].

Previously, we investigated the temperature dynamics during ultrafast laser microprocessing of glass by using time-resolved micro-Raman spectroscopy. We reported the spatial and temporal heat diffusion around the focal volume caused by the irradiation of low-repetition rate femtosecond pulses [3]. On the other hand, high-repetition-rate ultrafast laser pulses can induce smooth modifications and enable the usage of higher processing velocities [4-6]. Temperature dynamics is much more important therein because the processed area is strongly influenced by the heat accumulation effect, where successive pulses irradiate the sample before the induced heat diffuses out.

In this paper, we report our measurement of temperature dynamics inside glass by time-resolved micro-Raman spectroscopy during high-repetition-rate femtosecond laser microprocessing to investigate the temperature dynamics under the heat accumulation.

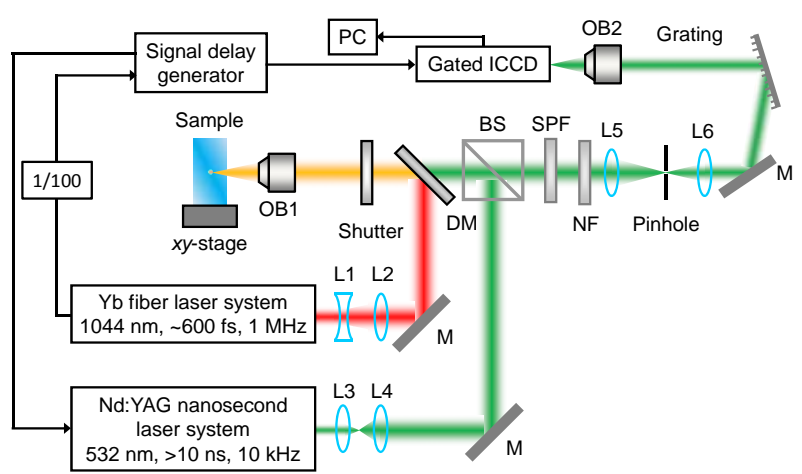

Fig. 1. Schematic of Raman temperature measurement system. OB1: Objective lens (20×, NA 0.46); OB2: Objective lens (5×, NA 0.13); L: Achromatic lens, L1: $\mathrm{f}=-75 \mathrm{~mm}, \mathrm{L2}: \mathrm{f}=100$ $\mathrm{mm}, \mathrm{L} 3: \mathrm{f}=30 \mathrm{~mm}), \mathrm{L} 4: \mathrm{f}=120 \mathrm{~mm}, \mathrm{L5}: \mathrm{f}=150 \mathrm{~mm}, \mathrm{L6}: \mathrm{f}=$ $100 \mathrm{~mm}$ ); SPF: $900 \mathrm{~nm}$ short pass filter; NF: $532 \mathrm{~nm}$ notch filter; M: Mirror; BS: Beam splitter; DM: Dichroic mirror; Gated ICCD: Gated intensified charge-couple-device. The diameter of the pinhole is $25 \mu \mathrm{m}$.

\section{Experimental setup}

A schematic of the Raman temperature measurement system is shown in Fig.1. For microprocessing, we used an amplified Ytterbium fiber laser system producing 600-fs pulses at a wavelength of $1044 \mathrm{~nm}$ and a repetition rate of 1 $\mathrm{MHz}$. For Raman pump, a frequency-doubled Nd:YAG laser was used to generate 10-ns pulses at a wavelength of $532 \mathrm{~nm}$ and a repetition rate of $10 \mathrm{kHz}$. The pulse energy of Raman pump pulse was set to $0.3 \mu \mathrm{J}$. These pulses were electrically synchronized with a pulse delay generator and then focused inside a sample $200-\mu \mathrm{m}$ below the surface by a $20 \times$ microscope objective lens with a numerical aperture of 0.46. As a sample, we used borofloat glass (Schott B33). 


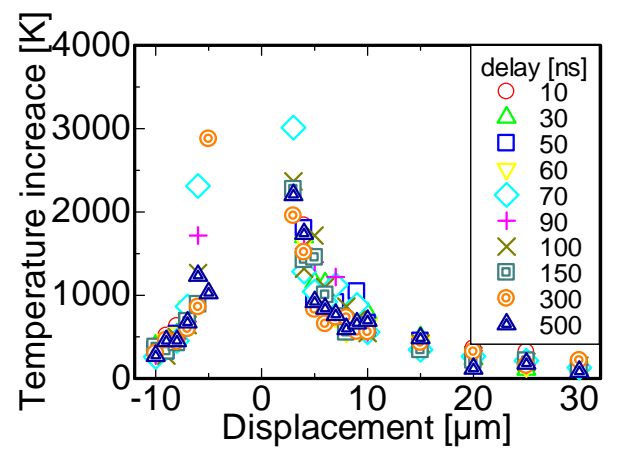

Fig. 2. The measured temperature distribution for a processing pulse energy of $1100 \mathrm{~nJ}$ and a scanning velocity of 10 $\mathrm{mm} / \mathrm{s}$.

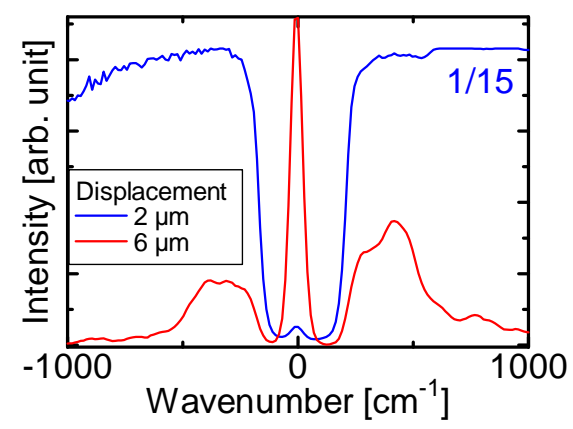

Fig. 3. Measured spectra for a displacement of 2 and $6 \mu \mathrm{m}$.

A computer-controlled $x y$-stage was used for raster scanning of the sample. By changing the scanning velocity, we controlled the number of irradiated pulses incident on the focal volume. The back-scattered Stokes and anti-Stokes Raman signals from the focus passed through a confocal pinhole and were detected by a gated intensified chargecoupled device (ICCD) camera, whose gating time was set to 10 ns. The gate of ICCD was synchronized with the Raman pump pulses. The time-gated Raman signal was accumulated 20000 times. With increasing temperature, mainly the intensity of the anti-Stokes Raman signal increases. Assuming a Boltzmann distribution of phonon population, the temperature can be estimated from the following equation [3]:

$$
T=-\left(\frac{h v_{R}}{k_{B}}\right) / \ln \left(\frac{I_{A S}}{I_{S}}\right),
$$

where $I_{\mathrm{S}}$ and $I_{\mathrm{AS}}$ are the intensity of Stokes and antiStokes Raman scattering, respectively, $h$ is the Planck constant, $v_{\mathrm{B}}$ is the Raman frequency, and $k_{\mathrm{B}}$ is the Boltzmann constant. Note that Eq. 1 works even for Raman spectra at high temperatures under the assumption that population of phonons obeys the Boltzmann distribution. Such assumption is reasonable considering that the typical phonon relaxation time is on the order of picoseconds, while we measure the temperature $10 \mathrm{~ns}$ after the irradiation. The emissivity of materials may change at high temperature, but its effect will be canceled out by calculating the ratio between Stokes scattering and anti-Stokes scattering. In order to measure the spatial distribution of temperature, the

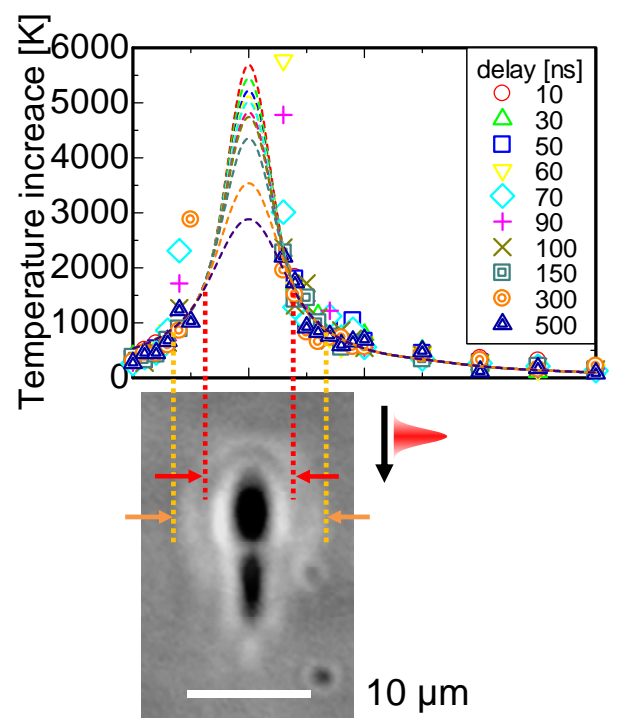

Fig. 4. Fitting results and the optical image of inscribed modification. The pulse energy of processing pulse was set to $1100 \mathrm{~nJ}$ and the scanning velocity was set to $10 \mathrm{~mm} / \mathrm{s}$.

focal position of the processing pulses was scanned perpendicularly to the scan direction of the sample. The temperature distributions around the focus along the line perpendicular to the scanning direction were measured. In this way, we measured the time-resolved temperature distribution during high-repetition-rate femtosecond laser processing.

\section{Results and Discussions}

\subsection{Measurement of temperature distribution}

Figure 2 shows the measured temperature distributions for a processing pulse energy of $1100 \mathrm{~nJ}$ and a scanning velocity of $10 \mathrm{~mm} / \mathrm{s}$. Within the range of $\pm 3 \mu \mathrm{m}$ from the center of the processing pulses, it was difficult to measure the temperature due to the onset of large background signals as shown in Fig. 3 (blue line). Nevertheless, we measured the temperature in the periphery of the focus of processing pulses as shown in Fig. 3 (red line). As shown in Fig. 2, we confirmed the decrease of the temperature depending on the position from the focus as expected. Note that the spatial distribution has broad tails and is different from a Gaussian profile, with which thermal diffusion occurs when the heat is deposited by single-shot irradiation [3]. This result suggests that the measured temperature is affected by the accumulation of heat by the successive processing pulses.

\subsection{Fitting curve of temperature distribution with heat accumulation effect}

In order to validate whether the origin of the difference from a Gaussian profile is the heat accumulation effect, the measured temperature data were fitted by the following equation [7]: 


$$
\begin{aligned}
T(t)= & A \sum_{n=0}^{N}\left\{\left(C \times w_{t h} / 2\right)^{2}+4 D_{t h}\left(t+n / f_{\text {rep }}\right)\right\}^{-1 / 2} \\
& \times\left\{\left(w_{t h} / 2\right)^{2}+4 D_{t h}\left(t+n / f_{\text {rep }}\right)\right\}^{-1} \\
& \left.\quad \times \exp L-(x-B)^{2} /\left\{\left(w_{t h} / 2\right)^{2}+4 D_{t h}\left(t+n / f_{\text {rep }}\right)\right\}\right\},
\end{aligned}
$$

where $w_{\text {th }}$ is the $1 / e$ width of the initial heated volume, $D_{\text {th }}$ is the thermal diffusivity of the material, $N$ is the number of pulses before the measured pulse arrives, $f_{\text {rep }}$ is the repetition rate of processing pulses (i.e. $f_{\text {rep }}=1 \mathrm{MHz}$ ), $t$ is the delay time, $A$ is a free fitting parameter including the energy absorptivity, $B$ is the spatial offset and $C$ is the aspect ratio of the initial heated volume. Assuming that the diameter of the initial heat is $2 \mu \mathrm{m}$ and that the scanning speed is $10 \mathrm{~mm} / \mathrm{s}$, we considered that the heat accumulation was caused by 200 pulses (i.e. $N=200$ ). Furthermore, we assumed that the heat source was elliptical, and neglected the dependences of the thermal diffusivity $\left(D_{\text {th }}\right)$ and the energy absorptivity $(A)$ on the temperature. Figure 4 shows the fitted curves assuming that $w_{\text {th }}, D_{\text {th }}, A, B$, and $C$ are constant for all delay times $t$. Especially in the outer region, the curves fit well. On the other hand, at the displacement smaller than $5 \mu \mathrm{m}$, the fitted curves yield slightly lower temperatures. The origin of this discrepancy could be the temperature dependence of $D_{\text {th }}$ and the energy absorptivity. Also the assumption of the ellipsoidal heat source might not be adequate $[8,9]$. Nevertheless, the presented results indicate the successful measurement of heat accumulation because the measured temperature fit well with Eq. (2), taking into account the heat accumulation of multiple successive pulses.

In order to check the validity of the fitting routine, we compared the fitting result with an optical image of the inscribed modification. The modification with the pulse energy of $1100 \mathrm{~nJ}$ and the scanning velocity of $10 \mathrm{~mm} / \mathrm{s}$ is shown in Fig. 4. From the optical image of the modification, we can see that the modification consists of two regions: one is the outer region, which has a bright contrast, and the other is the inner region, which has a dark contrast. From the comparison between the widths of the modification and temperature distribution, we can speculate that the outer region was formed in the range of temperature above $820 \mathrm{~K}$, which is the softening temperature of B33 glass. On the other hand, the inner region was formed in the range of temperature above $1270 \mathrm{~K}$, which is the working temperature of B33 [10]. In this way, the temperature distribution given by the fitting with Eq. 2 reasonably explains the resultant morphology of modifications.

\subsection{Dependence on pulse energy}

Figure 5 shows the maximal temperature increase induced with the various energies of the processing pulses. The scanning velocity was set to $10 \mathrm{~mm} / \mathrm{s}$. The temperature at the center for a delay time of $10 \mathrm{~ns}$ after the last pulse was extrapolated by the fitting. As shown in Fig. 5, the temperature increases by increasing the pulse energy. The temperature is highly sensitive to the incident pulse energy. This result indicates the need for the optimization of processing parameters when the high-repetition-rate ultrafast laser is used for material welding and the fabrication of photonic devices [11, 12].

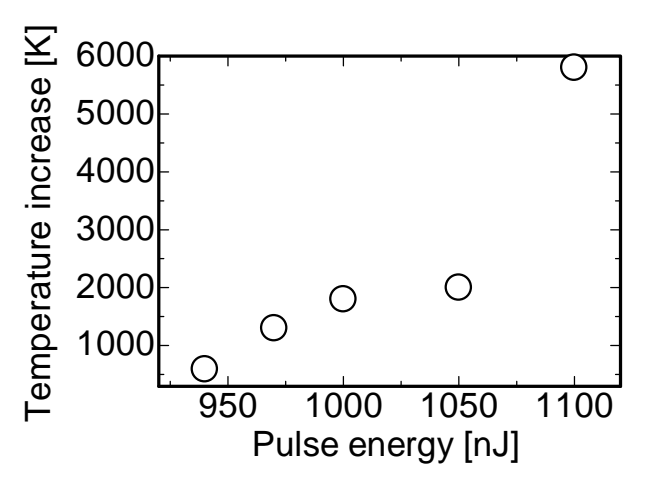

Fig. 5. Temperature dependence on pulse energy. Scanning velocity was set to $10 \mathrm{~mm} / \mathrm{s}$.

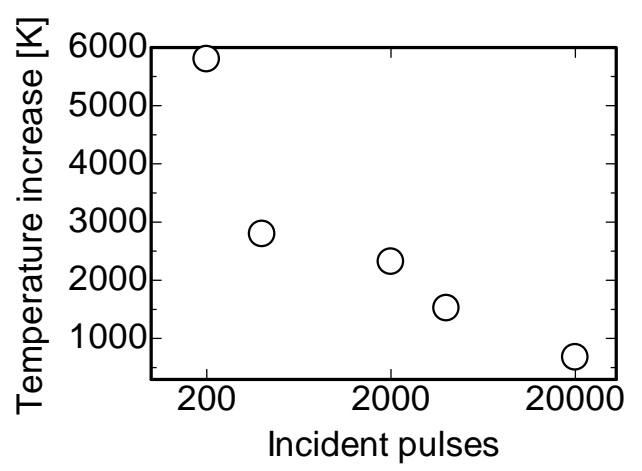

Fig. 6. Scanning velocity dependence of temperature distribution. Pulse energy was set to $1100 \mathrm{~nJ}$.

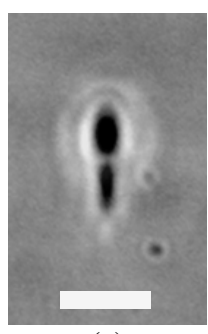

(a)

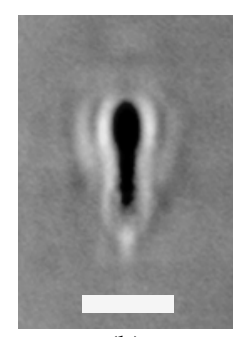

(b)

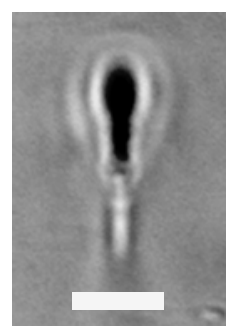

(c)
Fig. 7. Optical image of the inscribed modification with the scanning speed of (a) 10, (b) 1 and (c) $0.1 \mathrm{~mm} / \mathrm{s}$. The pulse energy was set to $1100 \mathrm{~nJ}$. Scale bar: $10 \mu \mathrm{m}$.

\subsection{Dependence on scanning velocity}

We further investigated dependence of the temperature on the scanning velocity, which is inversely proportional to the number of pulses irradiated to a certain region. Here, the scanning velocity is varied from 0.1 to $10 \mathrm{~mm} / \mathrm{s}$ (i.e. assuming the diameter of the initial heat of $2 \mu \mathrm{m}$, the effective number of pulses incident on a particular position $(N)$ is varied from 20000 to 200). The dependence of temperature on the effective number of incident pulses is shown in Fig 6. When a few hundred pulses are incident, the temperature at the center of the focal volume was estimated to be as high as $5000 \mathrm{~K}$. Unexpectedly, the temperature decreased as the number of incident pulses increased. In order to investigate this point, we observed the inscribed modifications by optical microscopy. Figure 7 shows the optical images of the processed regions. We can see that the modi- 
fied region elongates as the number of incident pulses increase. On the other hand, the widths of the modifications are not enlarged. Assuming that the outer modification is induced in the broad tails of the temperature distribution, caused by the heat accumulation effect, heat is not accumulated so much although the number of incident pulses was increased. These results suggest that the processing pulses are scattered by the inscribed modifications, leading to the temperature decrease at the center of modification. In this way, we conducted in situ investigation of the temperature dynamics and its dependence on processing parameters.

\section{Conclusion}

We have investigated temperature dynamics in femtosecond microprocessing of glass with high-repetition-rate femtosecond pulses. The measured temperature data were fitted taking the heat accumulation effect of successive pulses into account. The fitted results were in good agreement with the optical image of modification. We further investigated the influence of the pulse energy and the translation velocity on the temperature distribution. The latter result revealed the influence of structural modification on the processing temperature. The results of thermal process during the femtosecond laser microprocessing may provide useful information for its optimization, and it was confirmed that the time-resolved micro-Raman temperature measurement technique is a useful tool for in situ observation of the process.

\section{References}

[1] K. Itoh, W. Watanabe, S. Nolte, and C. B. Schaffer: MRS Bulletin, 31 (2006) 620.

[2] T. Tamaki, W. Watanabe, J. Nishii, K. Itoh, Japan. J. Appl. Phys. Part 2, 44 (2005) L687.

[3] T. Yoshino, Y. Ozeki, M. Matsumoto, and K. Itoh, Jpn. J. Appl. Phys., 51-10 (2012) 102403-5.

[4] C. B. Schaffer, J. F. Garcia and E. Mazur: Appl. Phys. A, 76 (2003) 351.

[5] S. M. Eaton, H. Zhang, P. R. Herman, F. Yoshino, L. Shah, J. Bovatsek and A. Y. Arai: Opt. Express, 13, (2005) 4708

[6] S. M. Eaton, H. Zhang, M. L. Ng, J. Li, W.-J. Chen, S.Ho and P. R. Herman: Opt. Express 16, (2008) 9443.

[7] M. Shimizu, M. Sakakura, M. Ohnishi, Y. Shimotsuma, T. Nakaya, K. Miura, and K. Hirao, J. Appl. Phys., 108 (2010) 073533-10.

[8] I. Miyamoto, K. Cvecek, and M. Schmidt, Opt. Express, 19 (2011) 10714.

[9] M. Shimizu, M. Sakakura, M. Ohnishi, M. Yamaji, Y. Shimotsuma, K. Hirao, and K. Miura, Opt. Express, 20 (2012) 934.

[10] http://www.valleydesign.com/Datasheets/Schott\%20B orofloat\%2033.pdf

[11] S. Richter, S. Döring, A. Tünnermann, and S. Nolte, Appl. Phys. A, 103 (2011) 257

[12] S. M. Eaton, M. L. Ng, R. Osellame, P. R. Herman, Journal of Non-Crystalline Solids 357 (2011) 2387.

(Received: July 21, 2013, Accepted: December 5, 2014) 